\title{
DEVELOPMENT OF RIVASTIGMINE LOADED SELF ASSEMBLED NANOSTRUCTURES OF NONIONIC SURFACTANTS FOR BRAIN DELIVERY
}

\author{
SARASWATHI T. S., MOTHILAL M.* \\ Department of Pharmaceutics, SRM College of Pharmacy, SRM Institute of Science and Technology, Kattankulathur, Tamil Nadu, India \\ Email: mothipharma78@gmail.com
}

Received: 07 Jul 2021, Revised and Accepted: 05 Aug 2021

\begin{abstract}
Objective: Aim of the study is to develop rivastigmine-loaded niosomal in situ gel via the intranasal route to the brain by crossing the Blood-Brain Barrier. For the treatment of Alzheimer's disease, it provides a speedy onset of action, a faster therapeutic effect, avoidance of the first-pass metabolism, and enhanced bioavailability.

Methods: Rivastigmine niosomal in situ nasal gel was developed, refined and tested with the goal of delivering the medicine to the brain via the intranasal route Rivastigmine niosomes were formulated by thin-film hydration technique, optimized using $\left(3^{2}\right)$ factorial design and characterized for its physicochemical parameters. Rivastigmine-loaded niosomes were further incorporated into Carbopal-934P and HPMC-K4M liquid gelling system to form in situ nasal gel. The resulting solution was evaluated for several parameters including, viscosity at pH 5 and pH 6 , gelling capacity
\end{abstract} and gelling time.

Results: Optimized best formulation containing span 60 (A) and cholesterol (B) with (1:0.5) ratio identified from the model developed from DesignExpert ${ }^{\circledR} 12$ software, exhibited Entrapment efficiency $(76.5 \pm 0.23 \%)$, particle size $(933.4 \pm 0.14$ nm), in vitro drug release maximum $(68.94 \pm 0.26 \%)$ at $8^{\text {th }}$ hour and further studied for its characteristics by SEM and TEM showed stable vesicles. Polynomial equations of Y1, Y2, and Y3 were conducted and ANOVA results showed a significant impact $(\mathrm{p}<0.05)$ on three levels. In vivo perfusion studies using rat model showed, the niosomes developed has good perfusion compared to pure drug with $27.2 \%$ of drug absorption in the brain at the end of 3 h. In vitro permeation of Rivastigmine through the dialysis membrane showed that $60.74 \% \mathrm{w} / \mathrm{w}$ drug permeated after 8 h. The formation of stable vesicles was proved by Zeta potential measurements and SEM analysis.

Conclusion: Optimized formulation had greater perfusion and was expected to have a good bioavailability compared to conventional other drug delivery systems.

Keywords: Niosomes, Blood-brain barrier, Factorial design, In situ gel, Rivastigmine, Intranasal

(c) 2021 The Authors. Published by Innovare Academic Sciences Pvt Ltd. This is an open access article under the CC BY license (https://creativecommons.org/licenses/by/4.0/)

DOI: https://dx.doi.org/10.22159/ijap.2021v13i5.42664. Journal homepage: https://innovareacademics.in/journals/index.php/ijap

\section{INTRODUCTION}

Alzheimer's disease (AD) is an advanced neurodegenerative condition of the brain that is the most common type of dementia, accounting for $60-80 \%$ of all dementia sufferers [1]. AD is the most common cause of cognitive impairment (memory, Intelligence level, and personality) in the elderly, and it is characterized by global cerebral cortex atrophy, relecting loss, and neuron shrinkage. $\mathrm{AD}$ is further distinguished by amyloid protein deposition in the form of senile plaque deposits and the creation of neurofibrillary tangles in the brain [2].

Nowadays, the nasal route for systemic medication delivery is an effective method for medicines with lesser oral-bioavailability due to presystemic intestinal and hepatic disposition. The concentrationtime profiles of medicines attained through nasal delivery are frequently identical to those obtained following intravenous injection resulting in a quick commencement of pharmacological activity [3] Intranasal administration is a practical method for delivering medications into the brain. Following nasal delivery around 35-40 substances including carbamazepine dopamine neurotoxic metals local anaesthetics, carboxylic acids and nerve growth factor have been shown to enter the central nervous system in experimental animals [4-9]. The nasal passages in the olfactory area have particular anatomic and physiologic properties that allow for both extracellular and intracellular pathways into the CNS, effectively bypassing the blood-brain barrier $[10,11]$.

Rivastigmine is a novel acetyl cholinesterase (AChE) inhibitor that is being used to treat Alzheimer disease. Rivastigmine inhibits AChE significantly more in the central nervous system (CNS) than in the periphery according to preclinical and clinical research. Rivastigmine is fast and completely absorbed (it absorbs more than 96 percent of the supplied amount). Peak plasma concentrations are obtained in about an hour and absolute bioavailability after a 3-mg dose is around
$36 \%$ Even though it is entirely absorbed extensive saturable first-pass metabolism reduces bioavailability. Rivastigmine is rapidly and extensively converted to the decarbamylated metabolite, principally by cholinesterase-mediated hydrolysis [12].

Carrier systems play a vital role in medication distribution into the system because they overcome nasal route secretions such as ciliary clearance breakdown by the nasal peptidase enzyme, and so on Niosomes, also known as non-ionic surfactant vesicles, are microscopic lamellar structures formed by combining an alkyl or dialkyl polyglycerol ether class non-ionic surfactant with cholesterol and then hydrating it in aqueous conditions [13]. One of the most beneficial properties of niosomes is their ability to direct medications to the intended site of action [14]. The nose-to-brain delivery route has the potential to be a less intrusive alternative to invasive techniques of medication delivery to the brain due to increased drug absorption and less systemic side effects [15].

The study's goal is to create Rivastigmine loaded niosomal in situ gel of diverse compositions. A 32 factorial design was used to assess the effect of formulation parameters such as surfactant type and cholesterol content ratio on the properties of niosomal vesicles. Furthermore the intranasal route of pharmaceutical administration is a favorable method of drug administration. Moreover the intra-nasal route of drug delivery is a promising method of drug delivery into the brain that might be enhanced by using appropriate carrier systems such as niosomes, therefore improving drug CNS penetration and delivering drugs in a sustained manner using an animal model.

\section{METHODS AND MATERIALS}

\section{Materials}

Rivastigmine was purchased from Orchid Orchid Pharma Ltd Tamilnadu, India. Cholesterol was from Himedia Labs Ltd., Tamilnadu, 
India. Sorbitan monostearate (Span 60) and methanol, chloroform from Himedia Labs Ltd., Tamilnadu, India. Carbopol-934 Analab Fine Chemicals Mumbai HPMC-K4M Sigma Aldrich Mumbai as well as other compounds collected from Loba Chemie Pvt. Ltd., Mumbai. All the other chemicals used in this research are of analytical grade.

\section{Methods}

\section{Formulation of rivastigmine-loaded niosomes}

Rivastigmine niosomes were made using a nonionic surfactant Span 60 using a lipid film hydration method. Different ratios of drug surfactant and cholesterol table 3 was precisely weighed and dissolved in a $15 \mathrm{ml}$ combination of chloroform and methanol $(2: 1 \mathrm{v} / \mathrm{v})$. The contents were evaporated for $30 \mathrm{~min}$ at a speed of 100 $\mathrm{rpm}$ in a Rotary evaporator at $60^{\circ} \mathrm{C}$ and a pressure of $25 \mathrm{~mm} \mathrm{Hg}$ to remove the solvent. The resultant film was hydrated with $10 \mathrm{ml}$ of pH 7.4 phosphate buffer saline. For $20 \mathrm{~min}$, a bath sonicator was used to sonicate the colloidal dispersion, and the niosomal solution was left overnight at $4{ }^{\circ} \mathrm{C}$ to develop $[16,17]$.

\section{Optimization and design of experiments}

To examine Rivastigmine loaded niosomal dispersions were generated under the combined impact of formulation factors utilizing a $3^{2}$-factorial design using Design-Expert $₫ 12$ Software. This design consists of two independent variables, Span 60 (A) and Cholesterol (B), which were analyzed in order to achieve optimum experimental efficiency with the fewest number of repetitions. The approach delivers a total of 9 experimental runs. The recognized dependent variables are Entrapment Efficiency (EE\%) (Y1), Particle size (Y2) and Maximum \% of drug release at $8^{\text {th }}$ hour (Y3).

\section{Statistical analysis of data}

The level of significance of the tested factors on the selected responses as well as the interaction between these factors were examined using Design-Expert $\circledast 8$ Software statistical tools which include descriptive statistics and one way ANOVA. A value of $\mathrm{p}<0.05$ was considered statistically significant.

\section{Characterization of drug loaded niosomal formulation}

\section{Entrapment efficiency}

The centrifugation method was used to determine the niosomal formulations percent entrapment efficiency ( $\%$ EE). For this experiment, $10 \mathrm{ml}$ of newly made niosomal formulations was placed in a centrifuge tube and centrifuged at $10000 \mathrm{rpm}$ at $4^{\circ} \mathrm{C}$ for $10 \mathrm{~min}$ using a cooling centrifuge (REMI cooling centrifuge). The supernatant was withdrawn and diluted with distilled water. A UV spectrophotometer was used to assess the concentration of free medicine in the supernatant layer (Shimadzu) and the entrapment efficiency of drug was calculated using the following equation

$$
\text { Entrapment Efficiency } \%=\frac{\text { Total drug }- \text { Free drug }}{\text { Total drug }} \times 100
$$

\section{Particle size}

The average diameter of sonicated vesicles was measured using laser diffraction method using a particle size analyzer (Horiba, SZ100, Japan).

\section{Scanning electron microscopy}

The niosomal vesicles morphology was analysed by a Scanning electron microscope (Jeol JSM 6510-USA).

\section{Zeta potential measurement}

The surface charge of Rivastigmine-loaded niosomal vesicles was measured using a Zeta potential analyzer (Horiba SZ-100). At $25^{\circ} \mathrm{C}$, the charge on the niosomes preparation with an average zeta potential were obtained in 60 seconds.

\section{Transmission electron microscopy (TEM)}

To analyze the morphology of the hydrated chosen niosomal dispersion (Model JEM-1230, Jeol, Tokyo, Japan), a drop of the dispersion was placed on a carbon coated copper grid and allowed to adhere for roughly $1 \mathrm{~min}$. A sheet of filter paper was used to eliminate the excess dispersion. To act as a negative staining agent, a drop of 1 percent phosphotungestic acid dispersion was used. The excess solution was collected using a tip of filter paper. Subsequently staining was carried out and samples were for drying at room temperature before being analyzed for $10 \mathrm{~min}$.

\section{Differential scanning calorimetry (DSC)}

Thermal properties were assessed using this device (Perkin Elmer 4000 , USA). The pure Rivastigmine, Span 60, and Cholesterol samples were weighed. Finally sealed in standard aluminum pans. Sample thermograms were acquired (at a scanning rate of 10 ${ }^{\circ} \mathrm{C} / \mathrm{min}$ ) in a temperature range of $30^{\circ} \mathrm{C}$ to $350^{\circ} \mathrm{C}$.

\section{$\mathrm{X}$-ray powder diffractometry (XRPD)}

X-ray crystallographic experiments were performed on Rivastigmine pure medication and its niosomal solution. An X-ray diffract meter (D8Advance BRUKER-Germany) with $2.2 \mathrm{KW}$ copper as anode material and an X-ray tube as a source was used to record the powder X-ray diffraction pattern. The material was examined with a lynux-eye detector then filtered with a Ni filter.

\section{In vitro drug release}

In vitro release studies were undergone using Dialysis bag diffusion technique. An accurately weighed amount of drug loaded niosomes containing the drug equivalent to $10 \mathrm{mg}$ was transferred to a dialysis bag and sealed. The sealed bag was then suspended in a beaker containing dialysis medium containing $250 \mathrm{ml}$ of phosphate buffer $\mathrm{pH} 7.4$ and stirred at a constant speed of 50rpm. Finally the drug content was determined using a UV/visible spectrophotometer set to $203 \mathrm{~nm}$ [18].

\section{In vivo nasal perfusion studies}

Using a rat model perfusion studies were utilized to investigate Rivastigmine absorption across nasal mucosa [19]. The in vivo nasal perfusion tests are carried out using Sprague dawley strain and male gender rats (Obtained from TANUVAS, Madhavaram, Chennai, Tamil Nadu) weighing about 200-300 g each, as described by Hussain et al. [20] and approved by Institutional Animal Ethical Committee (IAEC Registration number 662/PO/RE/S/2002/CPCSEA Dated on $14 / 02 / 2020$ ). The animals were acclimatized at $24 \pm 2{ }^{\circ} \mathrm{C}$ with a relative humidity. An intramuscular injection of ketamine hydrochloride and xylazine was used to anesthetize the rats, and an incision was made in the rat's neck. To prevent the rat from breathing by its nose the trachea was cannulated with a polyethylene tube. Another polyethylene tube was introduced through the oesophagus to the posterior parenchyma to allow the perfusion solution into the nostrils as shown in fig. 1.

To avoid thee drainage of the drug solution from the nasal cavity into the oral cavity, the nasopalantine region was plugged using an adhesive substance. By administering $5 \mathrm{ml}$ of normal saline through the oesophageal cannulation tube, the nasal cavity was washed. A persuate solution of niosomes with the known quantity was placed in a beaker and continually swirled with a magnetic stirrer and for the anaesthetized rat was then circulated through the nasal cavity.

The perfusing fluid was given to the nasal cavity by oesophageal cannulations and seeped from the nostrils, and was collected using a funnel and returned to the beaker. The rate of perfusion was kept constant at $4 \mathrm{ml} / \mathrm{min}$, and $1 \mathrm{ml}$ perfusate was taken at $1,2,3 \mathrm{~h}$ regular intervals and evaluated for drug content after replacing the fluid with fresh phosphate buffer saline of $\mathrm{pH} 7.4$. After $3 \mathrm{~h}$ the brain part of the rat model was isolated and minced with fresh phosphate buffer saline of $\mathrm{pH}$ 7.4 and then centrifuged, finally the filtrate was subjected to analysis.

\section{Preparation of niosomal in situ gel formulation}

Aqueous solutions of Carbopol-934P and HPMC-K4M in different concentrations were produced (table 8). Carbopol 934P was dispersed in water before gradually adding HPMC K4M as a viscosity modifier to the solution while stirring. This polymer combination was then treated with $10 \mathrm{ml}$ of prepared niosomal formulations (NR1) and $1 \%$ benzalkonium chloride. With distilled water the final volume was increased to $30 \mathrm{ml}$. A $0.5 \mathrm{M}$ sodium hydroxide solution 
was used to alter the $\mathrm{pH}$ of all of the sample solutions. Rivastigmine final concentration in the gel was 0.1 percent $\mathrm{w} / \mathrm{v}$.

To find compositions acceptable for use as in situ gelling systems, all prepared batches were assessed for gelling capacity, gelling time, and viscosity. The gelling capacity was calculated by inserting 100 of the composition (equal to $0.1 \mathrm{mg}$ of drug) in a vial containing $2 \mathrm{ml}$ of artificial nasal fluid (sodium $(150 \pm 32 \mathrm{mmol})$, potassium $(41 \pm 18$ mmol), calcium ( $4 \pm 2 \mathrm{mmol}$ ) and purified water q. $\mathrm{s} .100 \mathrm{ml}$ ) freshly prepared and equilibrated at $37^{\circ} \mathrm{C}$ and visually evaluated for gel formation. The time required for gelation and the time required for the gel produced to dissolve were both measured. The viscosity was measured at $15 \mathrm{rpm}$ using a Brookfield viscometer (DV Pro-II model with spindle no. 62.) in a small volume adapter [21, 22].

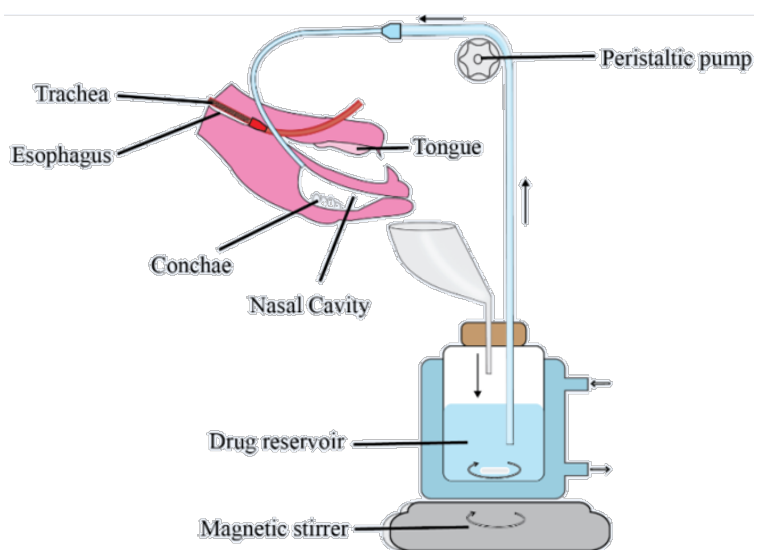

Fig. 1: In vivo nasal perfusion studies

\section{Characterization of niosomal in situ nasal gel formulation}

Table 8 shows the gelling time, gelling capacity, and viscosity results. The gelling capacity grade++was more adequate. Finally 0.2 percent Carbopol/0.6 percent HPMC was chosen as the recognized vehicle for further research.

\section{In vitro permeation studies}

Using a modified Franz diffusion cell across a dialysis membrane, an in vitro permeation analysis for the generated batches (ING1-ING9) was performed. The dialysis membrane was placed between the diffusion cell's donor and receptor compartments. The position of the donor compartment was adjusted so that the membrane barely touched the permeation medium.

A $2 \mathrm{mg}$ drug formulation was placed in the donor compartment, which was in direct contact with the membrane's mucosal surface, and the receptor compartment was filled with $20 \mathrm{ml}$ of PBS (pH 7.4) and kept at $37^{\circ} \mathrm{C}$. $1 \mathrm{ml}$ of sample was removed from the receptor compartment at a specified time point and replaced with the same volume of new medium [23]. The samples were evaluated for percent of drug penetrated from the formulations using a UV spectrophotometer at $203 \mathrm{~nm}$ after filtering through a $0.45 \mathrm{~m}$ filter and suitable dilution. A pure Rivastigmine loaded gel ( 0.1 percent $\mathrm{w} / \mathrm{v}$ RIV in Carbopol $934 \mathrm{P}$ ) was also made and subjected to permeation testing, with the results compared to the ING6 batch.

The cumulative amount of medication penetrated in $\mathrm{mg} / \mathrm{cm} 2$ was computed and plotted against time. The steady-state drug flux $(\mathrm{mg} / \mathrm{hr} / \mathrm{cm} 2)$ was estimated by dividing the slope of the linear component of the curve by the area of exposed tissue, and the permeability coefficient was obtained by dividing the flux by the initial drug load.

\section{RESULTS AND DISCUSSION}

\section{Selection of materials for the niosomal formulation}

Nonionic Surfactant and Cholesterol are key components in the production of niosomal vesicles, and their concentrations can impact particle size and entrapment efficiency.
Nonionic surfactants have a high interfacial activity and are composed of both polar and nonpolar regions. The surfactant's hydrophilic-lipophilic balance (HLB), the chemical structure of the components, and the critical packing parameter (CPP) are all important elements in the creation of bilayer vesicles. The size of the hydrophilic head group and the chain length of the nonionic surfactant have a significant impact on the drug's entrapment efficiency [24].

Ester-linked surfactants, also known as Spans, are polyoxyethylene esters derivatives that form less leaky vesicles that are more stable to osmotic gradients [25]. The length of the alkyl chain was also discovered to have a strong impact on entrapment efficiency; the longer the chain, the greater the encapsulation of the solute [26]. Because of its longer alkyl chain length, Span 60 vesicles have a greater entrapment efficiency [27]. More hydrophobic Spans form more compact niosomes when hydrated in the presence of cholesterol [28], which may allow for reduced drug diffusion to the release media [29].

Surfactants having the highest gel-to-liquid transition temperature $\left(\mathrm{T}^{\circ} \mathrm{C}\right)$ and longer alkyl chains, such as span 60 , have been shown to improve entrapment efficiency [30]. As a result, Span 60 was chosen and employed in this study. The hydrophilic-lipophilic balance value of the surfactant is influenced by the length of the alkyl chain, and the lower the HLB value, the better the entrapment efficiency. Span 60 entraps drug molecules without cholesterol by having a high phase transition temperature (gel to liquid transformation) and a critical packing parameter (CPP) in the $0.5-1$ range. The only disadvantage of Span 60 vesicles was rapid drug leakage due to the high phase transition temperature.

Cholesterol is the key component of a cell membrane that exhibits notable bilayer fluidity and permeability variations. To offer stiffness and orientational order, cholesterol is commonly included into non-ionic surfactants in large molar ratios. Cholesterol is the primary element that enhances bilayer membrane stability in the presence of biological fluids such as blood or plasma [31].

Cholesterol at a high concentration increases the stiffness of the bilayers by preventing the transformation of gel state into a liquid phase which results in the distruption of vesicular membrane structure. This has been related to ability of cholesterol to boost phospholipid membrane packing density [32], which is assumed to be due to cholesterol accommodation within molecular cavities generated by surfactant monomers packed into vesicles. In this niosomal formulation cholesterol inclusion aids to rise the overall CPP of the system,

Because cholesterol intercalation inside the system would be deleterious, lowering the average area per molecule and lowering the total CPP, the presence of cholesterol in this niosomal formulation serves to raise the overall CPP of the system.

\section{Analysis of factorial design}

Extensive literature research and formulation studies are necessary for the development and selection of niosomal dispersions. The surfactant used, its concentration, and the amount of cholesterol supplied can all alter the features of the niosomes generated, hence these are all regarded critical variables in achieving a stable and effective niosomal system. The physicochemical properties of Rivastigmine loaded niosomes obtained from the experimental design were analyzed. Table 1 shows the coded and actual values for the independent factors. Table 2 and table 3 shows the experimental runs with the specified variables and their effects on the measured responses.

\section{Design of experiments}

The responses were evaluated using the following statistical model, which included interactive and polynomial terms:

$$
Y=\beta_{0}+\beta_{1} A+\beta_{2} A+\beta_{3} A B+\beta_{4} A^{2}+\beta_{5} B^{2}+\beta_{6} A B+\beta_{7} A B^{2}
$$

Where $\mathrm{Y}$ is the dependent variable, $?_{0}$ is the arithmetic mean response of the 9 runs and $B 1$ is the estimated coefficient for the factor $A$. The average result when the components were modified one at a time from low to high values is shown by the primary effects 
of the amounts of $A$ and $B$. The interaction terms (AB) show how the response changes when two factors are simultaneously changed. The results show that particle size and entrapment efficiency are highly influenced by independent factors used [33].

In this experimental design totally 9 runs were suggested by the $3^{2}$ factorial design for two independent variables: Span 60 and Cholesterol that were at three different levels (high, medium and low). The ANOVA results as illustrated in table 4 table 5 and table 6 showed that all models were significant $(p<0.05)$. Models were simplified by removing non-significant terms $(p>0.05)$ from equations resulting in:

The model equation relating Particle size as response became:

$$
Y=\beta_{0}+\beta_{1} A+\beta_{2} B+\beta_{3} A B
$$

Particle size $(\mathrm{nm})=1096.03-184.35(\mathrm{~A})+157.783(\mathrm{~B})-254.875(A B)$

The model equation relating $\% \mathrm{EE}$ as response became:

$$
Y=\beta_{0}+\beta_{1} A+\beta_{2} B
$$

$\mathrm{EE} \%=78.1411-7.865(\mathrm{~A})+18.97(\mathrm{~B})$

The model equation relating $\%$ DR Max at $8^{\text {th }} \mathrm{h}$ as response became:

$$
Y=\beta_{0}+\beta_{1} A+\beta_{2} B+\beta_{3} A B
$$

Max \% DR at $8^{\text {th }} \mathrm{h}=49.7222-6.36333(\mathrm{~A})+2.295(\mathrm{~B})+9.245(\mathrm{AB})$

The above given model equation, confirms that the positive sign of the factors indicates the beneficial or synergistic interaction towards the responses, whereas negative sign indicates less or no significance toward the responses.

Table 1: Factors for designing of formulations

\begin{tabular}{lll}
\hline Factors & Coded values & Actual values $\left(\times \mathbf{1 0}^{-2} \mathbf{M}\right)$ \\
\hline Span 60 (Factor A) & -1 & 1 \\
& 0 & 2.5 \\
Cholesterol (Factor & +1 & 4 \\
B) & 0 & 0.5 \\
& +1 & 1.25 \\
\hline
\end{tabular}

Table 2: Recommended formulations containing span 60 and cholesterol as per DOE

\begin{tabular}{lll}
\hline Batch No & Span 60 & Cholesterol \\
\hline 1 & -1 & -1 \\
2 & -1 & 0 \\
3 & -1 & +1 \\
4 & 0 & -1 \\
5 & 0 & 0 \\
6 & 0 & +1 \\
7 & +1 & -1 \\
8 & +1 & 0 \\
9 & +1 & +1 \\
10 & 0 & 0 \\
11 & 0 & 0 \\
\hline
\end{tabular}

Table 3: Composition of rivastigmine loaded niosome by $3^{2}$ factorial design-response parameters

\begin{tabular}{llll}
\hline Formulation batch code & Entrapment efficiency (\%) Y1 & Particle size (nm) Y2 & Maximum \% drug release at 8 ${ }^{\text {th }} \mathbf{h}$ 3 \\
\hline NR1 & $76.5 \pm 0.23$ & $933.4 \pm 0.14$ & $68.94 \pm 0.26$ \\
NR2 & $90.37 \pm 0.14$ & $1158.8 \pm 0.22$ & $54.31 \pm 0.14$ \\
NR3 & $94.46 \pm 0.43$ & $1678 \pm 0.31$ & $51.27 \pm 0.16$ \\
NR4 & $45.71 \pm 0.15$ & $759.7 \pm 0.66$ & $40.65 \pm 0.66$ \\
NR5 & $89.09 \pm 0.42$ & $1433.6 \pm 0.54$ & $43.21 \pm 0.28$ \\
NR6 & $93 \pm 0.43$ & $1236.7 \pm 0.48$ & $52.78 \pm 0.21$ \\
NR7 & $42.86 \pm 0.28$ & $981 \pm 0.36$ & $35.41 \pm 0.34$ \\
NR8 & $79.85 \pm 0.16$ & $977 \pm 0.87$ & $46.21 \pm 0.36$ \\
NR9 & $91.43 \pm 0.18$ & $706.1 \pm 0.94$ & $54.72 \pm 0.22$ \\
\hline
\end{tabular}

mean $\pm \mathrm{SD}(\mathrm{n}=3)$

\section{Particle size}

The average vesicle diameter after being sonicated was measured using laser-diffraction method and the all niosomal formulation batches have vesicle sizes ranging between 706.1-1433.6 nm (table 3).

Span 60, and Cholesterol have been fixed at low 706.1 and high 1678 based on the results of the prediction profiler.

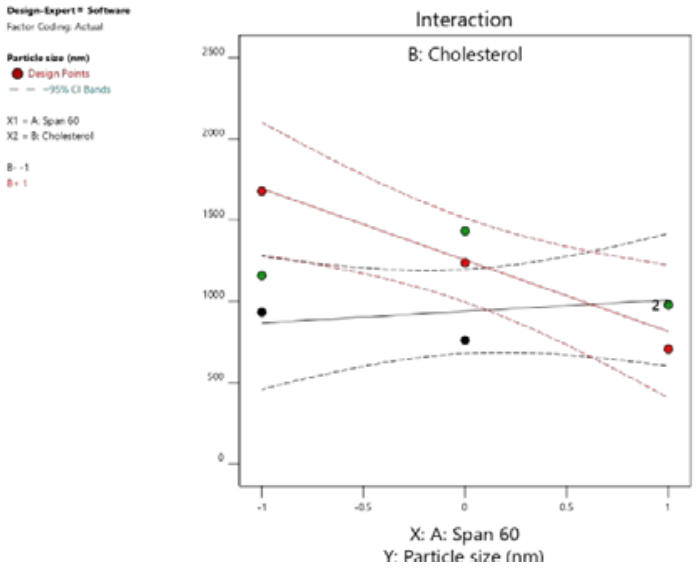

Fig. 2: Effect of factor $A$ and $B$ on particle size
Interaction graph shows a significant interaction between the independent factors (Variables) A and B. As shown in ANOVA table 4 the Model F-value of 5.61 implies that the model is significant. P-values less than 0.0500 indicate model terms are significant. Thus it is confirmed that in this case $\mathrm{AB}$ is a significant model term and independent factors have relationship with the response (particle size) (fig. 2).

\section{Entrapment efficiency}

By using Centrifugation method, Entrapment efficiency of the formulations were done. The remaining predictors are fixed at a given point. Span 60 , and Cholesterol have been fixed at low 42.86 and high 94.46 based on the results of the prediction profiler.

ANOVA table 5 shows that the Model F-value of 13.36 implies the model is significant. P-value is 0.0291 which is less than 0.0500 indicates that model terms are significant. In this case B is a significant model term. It is constructed by predicting the responses for the low (1 ) and high (+1) levels of a factor. The interaction graph shows clearly that there is a significant interaction between Span 60 (A) and Cholesterol (B) and thus it confirms the significant changes in the response. The contour-lines represent lines of equal response and can be visualized as response contours (two factors at a time).

\section{In vitro drug release}

The cumulative in vitro drug release was performed in phosphate buffer $\mathrm{pH} 7.4$ by dialysis method. The remaining predictors are fixed at a given point. Span 60, and Cholesterol have been fixed at low 35.41 and high 68.94 based on the results of the prediction profiler. 
ANOVA table 6 shows that the Model F-value of 6.73 implies the model is significant. P-value is 0.033 which is less than 0.0500 indicates that model terms are significant. In this case $A B$ is a significant model term. Interaction graph confirms that the independent factors involve that $\mathrm{AB}$ interaction (fig. 4). The contourlines represent lines of equal response and can be visualized as response contours (three factors at a time).

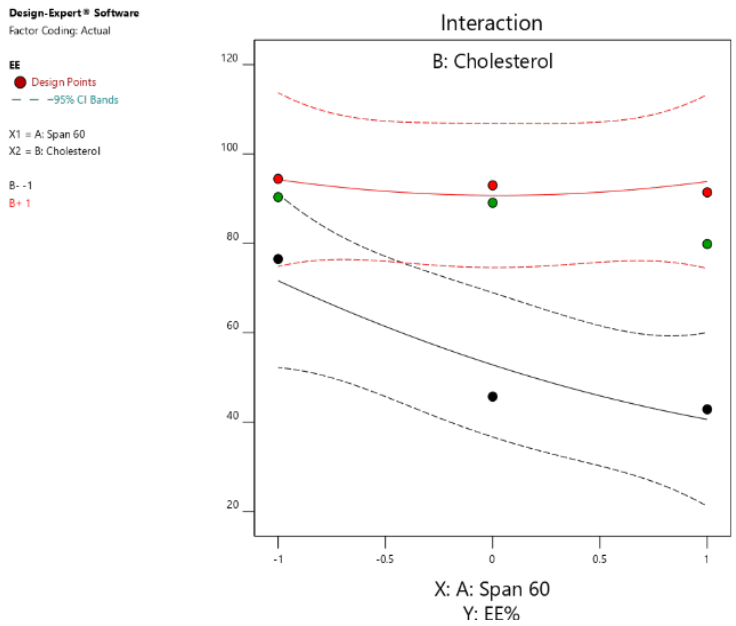

Fig. 3: Effect of factor $A$ and B on entrapment efficiency

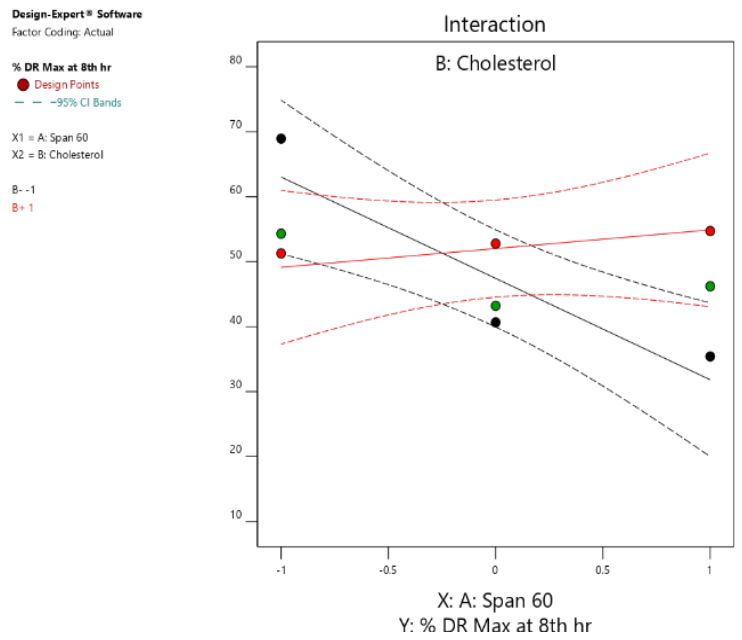

Fig. 4: Effect of factor $A$ and $B$ on in vitro drug release
Effect of surfactant concentration on particle size and entrapment efficiency

Surfactant is a key component in the production of niosomal vesicles and its concentration can influence particle size as well as entrapment efficiency. It was revealed in this investigation that increasing the concentration of Span 60 increases particle size. This can be seen on changing the concentration of Span 60 from-1 level to 0 level. From the results, there is a significant increase in the entrapment efficiency was observed for-1 level of Span 60 however on further increase in the level of Span 60 from-1 to 0 level the entrapment efficiency decreases.

The number of niosomes formed increases with initial increase in the concentration of surfactant; consequently the volume of hydrophobic domain increases and hence increases in entrapment efficiency. Further increase in surfactant concentration caused a decline in entrapment efficiency, apparently due to the development of mixed micelles in association with niosomal vesicles containing high surfactant concentrations, which could lead to a reduction in entrapment efficiency [34].

\section{Effect of cholesterol on particle size and entrapment efficiency}

The amount of cholesterol in the vesicles and the particle size of the vesicles both play a role in drug entrapment. Both the variables viz. particle size and entrapment efficiency are affected by variations in cholesterol content. An increase in cholesterol concentration causes an increase in particle size.

A higher entrapment efficiency was found on increasing concentration of cholesterol from-1 to 0 level. Cholesterol, when present in high concentrations, prevents the gel state from transforming into a liquidordered phase, increasing the stiffness of the resultant bilayers [35].

Thus it has been concluded that a small concentration of cholesterol at-1 level is optimum to get stable vesicles by abolishing the phase transition temperature and avoiding drug leakage according to $\mathrm{S}$. Srinivas et al. 2010 [36]. Thus, the cholesterol in these formulations was found to prevent the rapid leakage of drug from vesicles and NR1 formulation containing Span 60 Cholesterol in the ratio of 1:0.5 is found to be optimum to get stable vesicles.

From the observation it was concluded that NR1 (Span60: CH 1:0.5), the selected niosomal formulation exhibited high drug EE\%, small PS and higher percentage of drug release at $8^{\text {th }}$ hour having spherical shaped vesicles.

NR1 (Span 60 (A): Cholesterol (B)) 1: 0.5-(EE\%) 76.5\%; (PS) 933.4 $\mathrm{nm}$; (\% drug Release at $\left.8^{\text {th }} \mathrm{h}\right) 68.94 \%$.

Analysis of variance (ANOVA), applied at a 5\% significance level, indicated that the postulated regression model was statistically significant and valid as shown in table 4 and table 7 . This also proved that type of Span 60: Cholesterol ratio and their interaction have a significant effect on the EE\% of Rivastigmine niosomes and Particle size as well as Drug release at $8^{\text {th }}$ h. Fig. 5, fig. 6 and fig. 7 represents the surface response plot showing relative effect of different process parameter on Particle size, Entrapment Efficiency and Drug release at $8^{\text {th }} \mathrm{h}$.

Table 4: Analysis of variance for particle size (nm)

\begin{tabular}{|c|c|c|c|c|c|}
\hline Source & Sum of square & df & Mean square & F-value & P-value \\
\hline \multicolumn{6}{|c|}{ Particle size (nm) (2FI)-Significant } \\
\hline Model & 695159.3 & 3 & 231719.8 & 5.61 & $0.046^{*}$ \\
\hline A-Span 60 & 200604.7 & 1 & 200604.7 & 5.59 & 0.0643 \\
\hline B-Cholesterol & 234709.5 & 1 & 234709.5 & 4.10 & 0.0988 \\
\hline $\mathrm{AB}$ & 259845.1 & 1 & 259845.1 & 7.13 & 0.0444 \\
\hline
\end{tabular}

$\mathrm{R}^{2}$ Value -0.77

Table 5: Analysis of variance for \% entrapment efficiency

\begin{tabular}{|c|c|c|c|c|c|}
\hline Source & Sum of square & df & Mean square & F-value & P-value \\
\hline \multicolumn{6}{|c|}{ EE \% (Quadratic)-Significant } \\
\hline Model & 3096.17 & 5 & 619.23 & 13.36 & $0.0291^{*}$ \\
\hline A-Span 60 & 371.15 & 1 & 371.15 & 8.01 & 0.0662 \\
\hline B-Cholesterol & 2159.17 & 1 & 2159.17 & 46.57 & 0.0064 \\
\hline $\mathrm{AB}$ & 234.24 & 1 & 234.24 & 5.05 & 0.1102 \\
\hline $\mathrm{A}^{2}$ & 21.93 & 1 & 21.93 & 0.4731 & 0.5410 \\
\hline $\mathrm{B}^{2}$ & 309.67 & 1 & 309.67 & 6.68 & 0.0815 \\
\hline
\end{tabular}

$\mathrm{R}^{2}$ Value-0.95 
Table 6: Analysis of variance for $\%$ drug release maximum at $8^{\text {th }} \mathbf{h}$

\begin{tabular}{|c|c|c|c|c|c|}
\hline Source & Sum of square & df & Mean square & F-value & P-value \\
\hline \multicolumn{6}{|c|}{$\%$ DR at $8^{\text {th }}$ hr $(2 \mathrm{FI})-$ Significant } \\
\hline Model & 616.43 & 3 & 205.48 & 6.73 & $0.033^{*}$ \\
\hline A-Span 60 & 242.95 & 1 & 242.95 & 7.96 & 0.0370 \\
\hline B-Cholesterol & 31.60 & 1 & 31.60 & 1.04 & 0.3555 \\
\hline $\mathrm{AB}$ & 341.88 & 1 & 341.88 & 11.21 & 0.0204 \\
\hline
\end{tabular}

$\mathrm{R}^{2}$ Value- 0.80

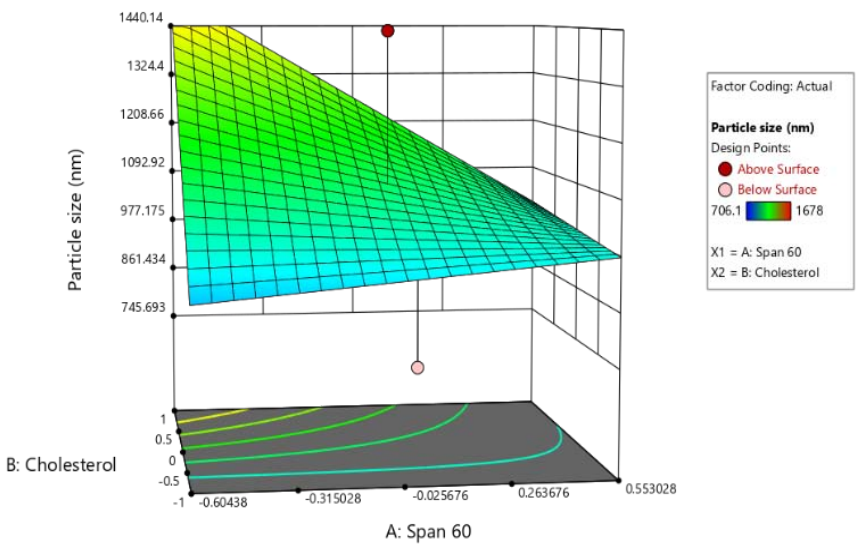

Fig. 5: 3D Surface response plot showing relative effect of different process parameter on particle size
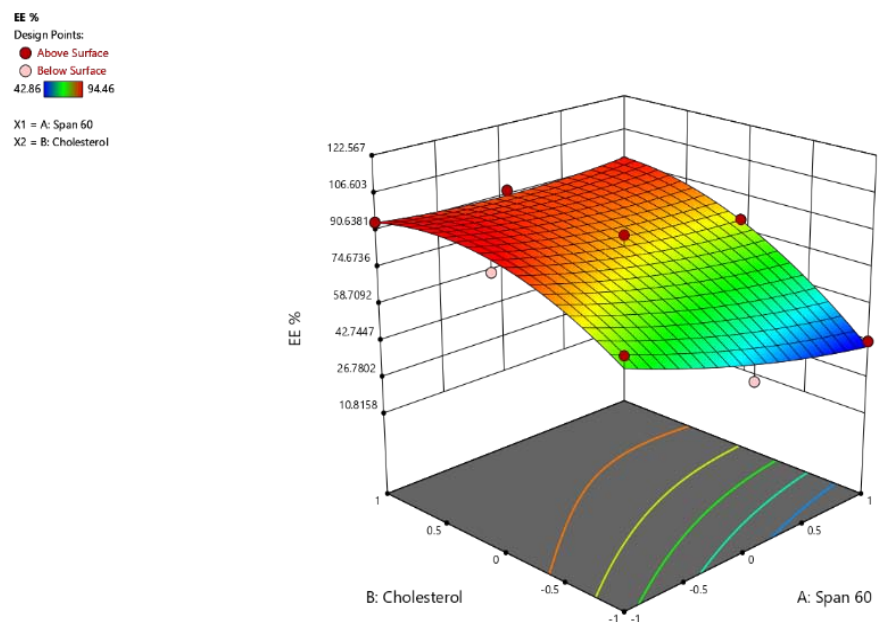

Fig. 6: 3D Surface response plot showing relative effect of different process parameter on entrapment efficiency

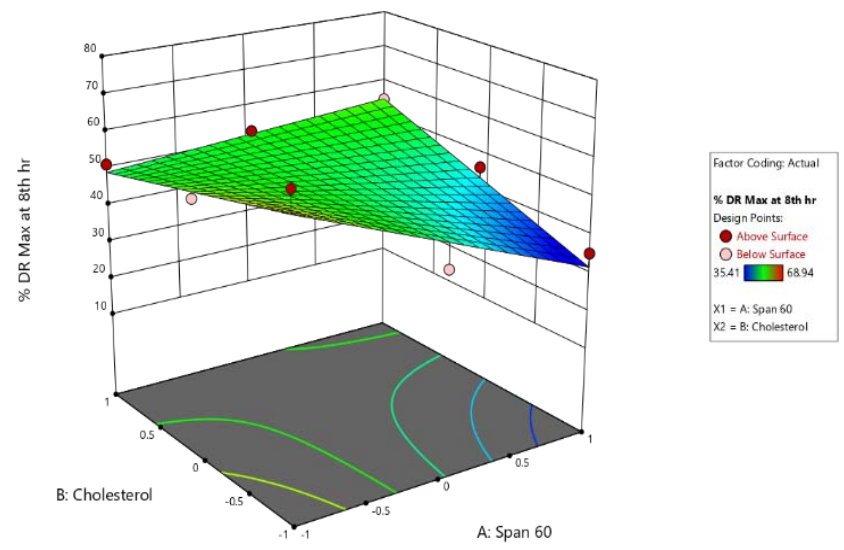

Fig. 7: 3D Surface response plot showing relative effect of different process parameter on drug release at $8^{\text {th }} h$ 
Table 7: Point prediction

\begin{tabular}{lll}
\hline Response & Predicted mean & Observed \\
\hline EE \% & 84.2289 & $84.1232 \pm 0.90$ \\
\% DR Max at 8th h & 49.7222 & $50.4543 \pm 1.02$ \\
Particle size (nm) & 1096.03 & $1098.31 \pm 2.09$ \\
\hline
\end{tabular}

mean \pm SD $(n=3)$

\section{Validation and optimized formulation}

An optimized formula of Rivastigmine loaded niosomes which fulfil the requirement of achieving maximum entrapment efficiency, maximum drug release at 8th hour and minimum vesicle size is chosen for further study. The improved formulation is generated and analysed to validate the computed optimal factor levels and their predicted responses. From the results table 7 it can be concluded that optimized formula showed vesicle size, entrapment efficiency and drug release profile as predicted from the model equations developed by the present study without significant difference.

The vesicle size of all niosomal formulations batches ranges between 706.1-1433.6 nm (table 3). The vesicle size of NR1 batch is $933.4 \mathrm{~nm}$ (fig. 8). A scanning electron microscope was used to examine the morphology of the synthesised niosomal formulation. The value of zeta potential was found to be-15.4 mV for optimized niosomal formulation (NR1) (fig. 9). It stipulates that produced niosomes have a sufficient surface charge to prevent vesicle aggregation.

The SEM of optimised niosomal formulation (NR1 batch) exhibits spherical dark stained niosomal particles in the nanosize range, with a particle diameter of less than $1 \mu \mathrm{m}$ (fig. 10).

The morphology of Rivastigmine-loaded niosomes was studied using Transmission electron microscopy (TEM). Fig. 11 shows the micrographs of NR1 show well-identified vesicles in a nearly perfect sphere-like shape with a smooth surface. The diameters of the vesicles detected in the micrographs were consistent with particle size analysis data.

Differential scanning calorimetry (DSC) is a technique for determining the drug's thermal behaviour and physical condition, as well as any potential interactions with other substances. The melting point of Rivastigmine was reported to be $125.71{ }^{\circ} \mathrm{C}$. Span 60 and cholesterol showed a sharp endothermic peak at 53.62 and $141.1^{\circ} \mathrm{C}$, respectively. The melting point of both the excipients was as shown in the two peaks i.e. $54-57^{\circ} \mathrm{C}$ and $148-150{ }^{\circ} \mathrm{C}$. From the results it was concluded that all other excipients (Span 60 and Cholesterol) were of optimum suitability because of their compatibility with the drug as shown in fig. 12 .

Rivastigmine demonstrated a strong and distinct XRD pattern consistent with crystalline powder, whereas formulation NR1 exhibited peak loss and more diffuse peaks (fig. 13). This confirms that the crystalline form of the Rivastigmine changed into amorphous form.

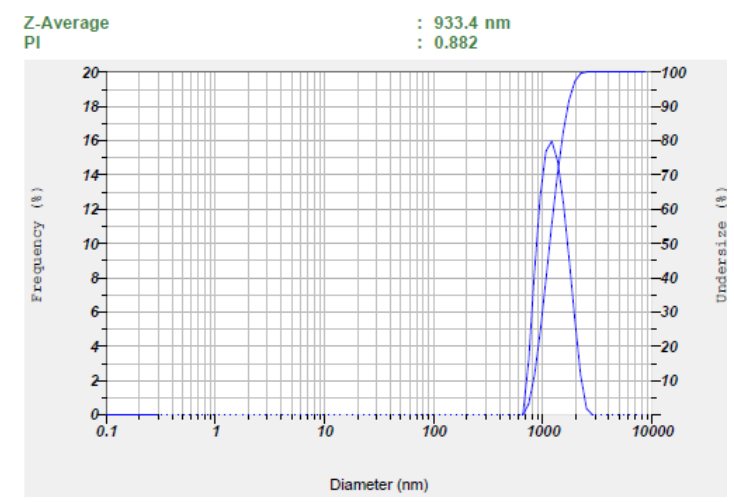

Fig. 8: Particle size

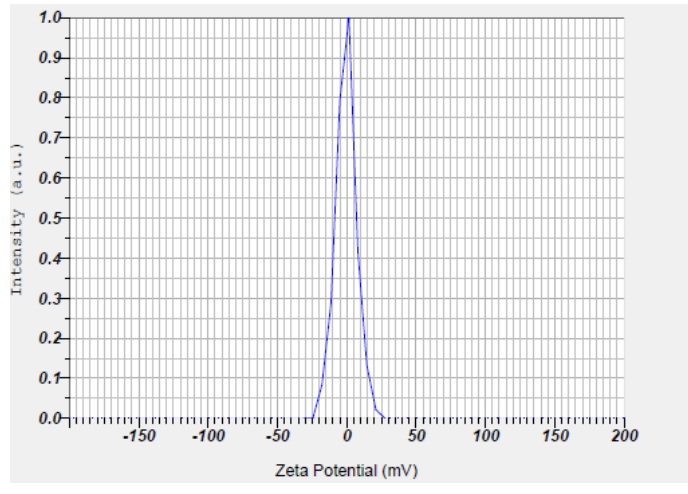

Fig. 9: Zeta potential

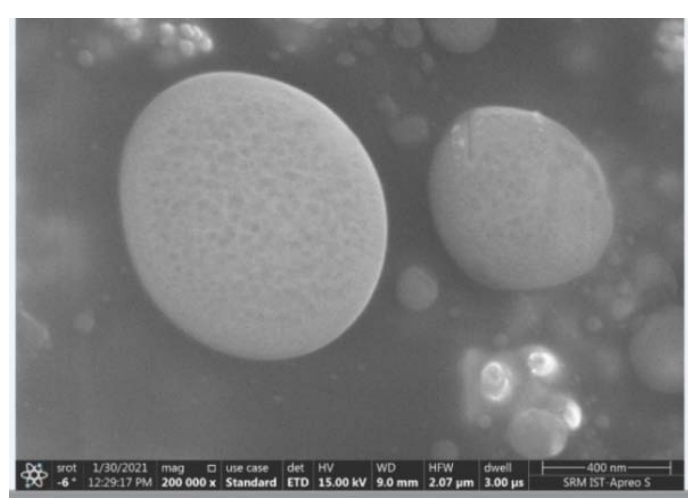

Fig. 10: FE-SEM image

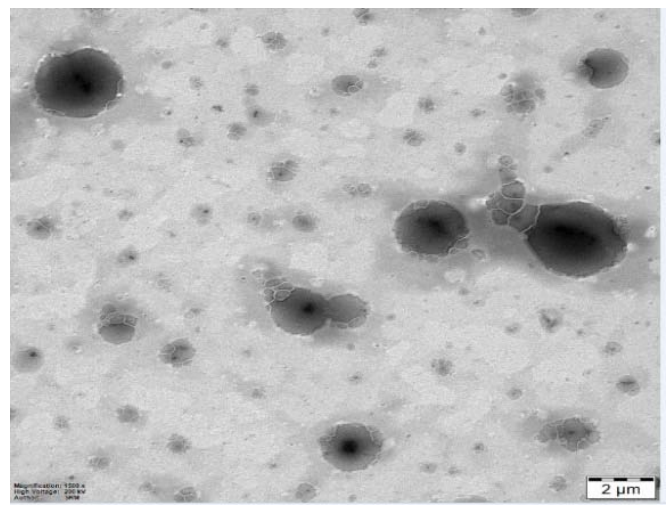

Fig. 11: TEM image

\section{In vitro drug release kinetics}

Fig. 14 shows the percent cumulative release of niosomal formulation compositions NR1-NR9. The cumulative percent release of niosomal formulations ranges from" $35.41 \%$ to $68.94 \%$.

Kinetics of release of the optimized formulation (table 8) followed zeroorder kinetics. Higuchi's correlation coefficient showed that the drug release is proportional to the square root of time indicating that Rivastigmine release is diffusion controlled. The $n$ value from the korsemeyer Peppas model for Rivastigmine loaded niosomes was 0.833 which confirms the non-Fickian diffusion or anomalous transport.

\section{In vivo nasal perfusion studies}

Compared to the pure drug, about $27.2 \%$ of Rivastigmine loaded niosomes was better absorbed from the nasal cavity at the end of 3 $\mathrm{h}$, which confirms that the optimized niosomal batch NR1 can be useful for targeting the brain (fig. 15). 


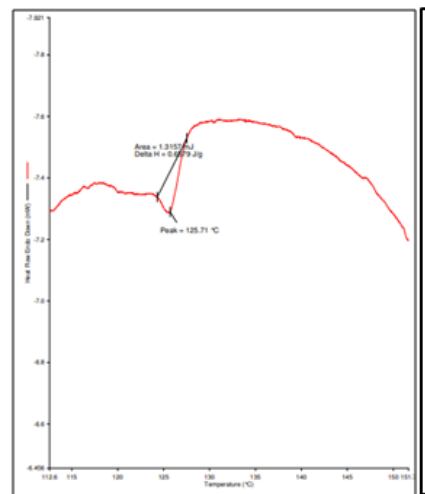

(a)

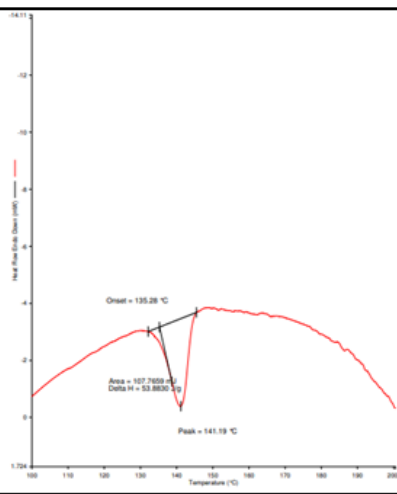

(b)

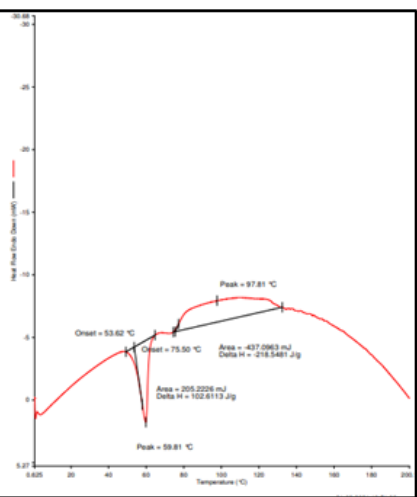

(c)

Fig. 12: DSC Thermograms of a) Rivastigmine b) Rivastigmine with cholesterol c) rivastigmine with span 60

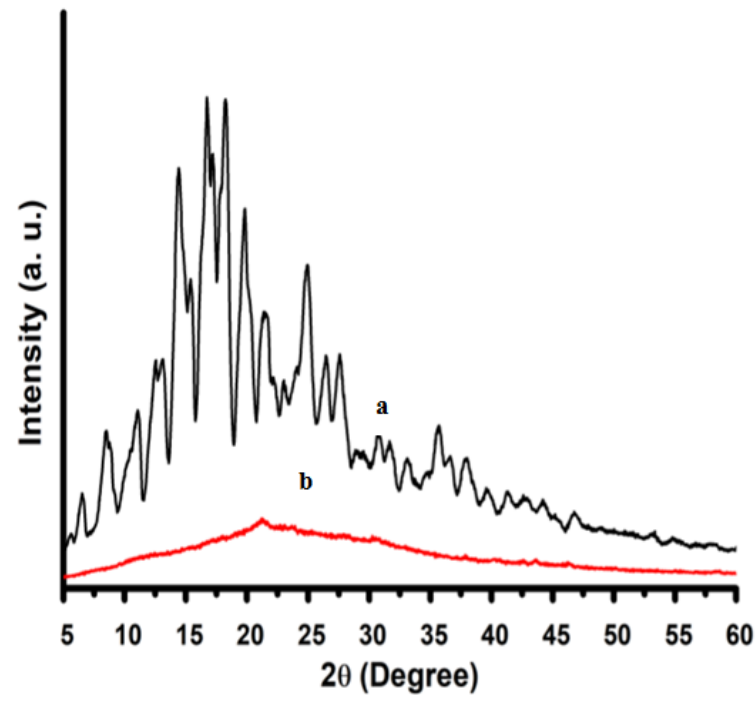

Fig. 13: XRD patterns of a) Rivastigmine pure drug b) Rivastigmine niosomes

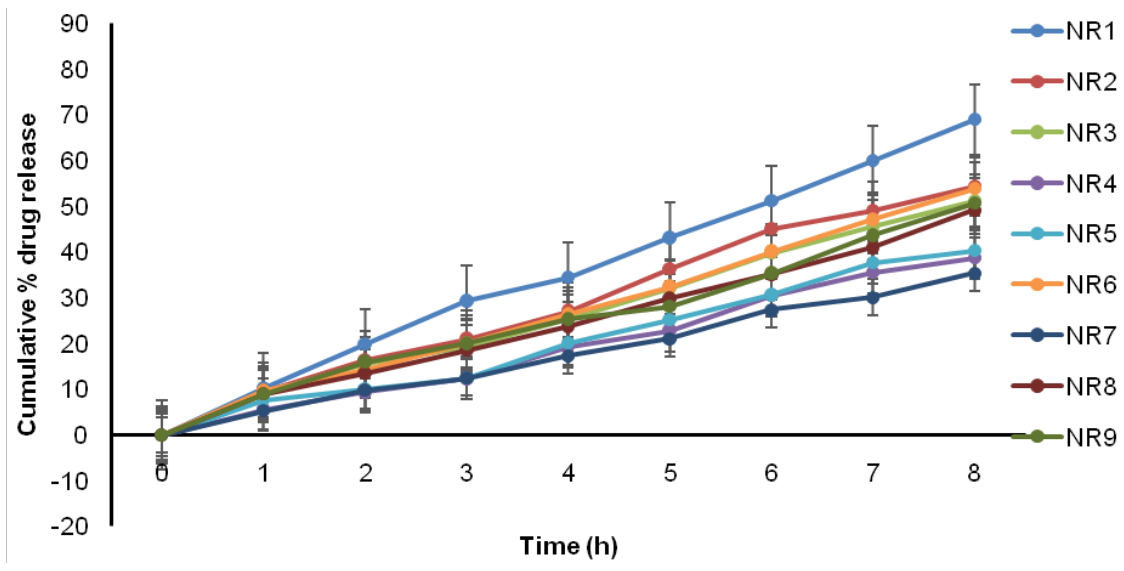

Fig. 14: Cumulative percentage release of niosomal formulation batches NR1-NR9

Table 8: Release kinetics of optimized rivastigmine loaded niosomal formulation (NR1)

\begin{tabular}{lllllll}
\hline Formulation & Higuchi & Korsemeyer peppas & Zero order & First order & Hixson-crowell \\
\cline { 2 - 6 } & $\mathbf{r}^{\mathbf{2}}$ & $\mathbf{n}$ & $\mathbf{r}^{\mathbf{2}}$ & $\mathbf{r}^{\mathbf{2}}$ & $\mathbf{r}^{\mathbf{2}}$ & \\
\hline NR1 & 0.9791 & 0.833 & 0.9974 & 0.997 & 0.973 \\
\hline
\end{tabular}




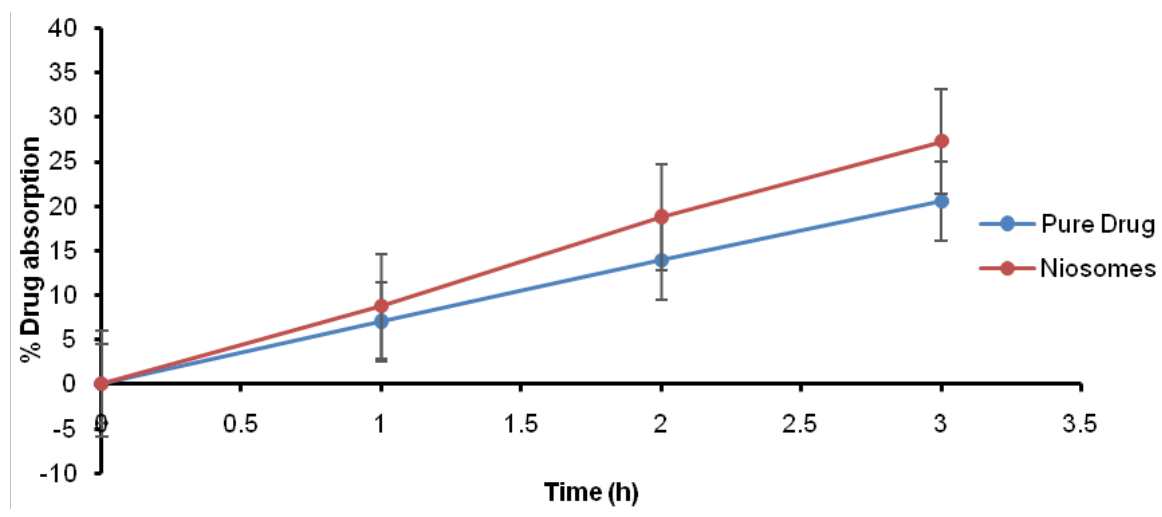

Fig. 15: In vivo nasal absorption profile of pure drug and niosomal formulation NR1

\section{Preparation of niosomal in situ gel formulation}

Reversibly gelling compositions can be manufactured at low polymer concentrations utilising two or more suitable polymers, one of which is sensitive to $\mathrm{pH}$ changes while the other does not need to be sensitive to temperature changes. Lowering the buffering capacity and heat gelation threshold can be achieved by utilizing comparatively low concentrations of polymeric components. This leads to the rapid development of better gels in-situ and the reduction of patient discomfort caused by prolonged exposure of tissues to $\mathrm{pH}$ extremes. Because less polymer is used, the cost of producing such formulations is likewise decreased. Furthermore, these beneficial properties are achieved using a polymeric formulation whose gelation is driven by only one physicochemical parameter (i.e., pH change) [37].

The ability of Carbopol aqueous solutions to change into stiff gels when the $\mathrm{pH}$ is raised justifies its usage in in situ gelling systems [38,
39]. The inclusion of polymers improving the viscosity for instance HPMC can reduce Carbopol content without impairing the gelling capacity and rheological properties of the delivery system. Different concentrations of Carbopol 934P and HPMC K4M were made and analysed for gelling capacity in order to recognise the compositions suited for use as in situ gelling systems, as indicated in table 9. The viscosity and gelling capability of an in-situ gelling system are the two most important factors to consider. The formulation should have a viscosity that allows for simple instillation as liquid drops into the nasal canal, wherein there exhibits a quick sol-to-gel transition (triggered by a rise in $\mathrm{pH}$ from 5.0 to 6.0).

The developed in situ nasal gel niosomal formulation (ING9) containing ( 0.2 percent Carbopol/0. 6 percent HPMC) was chosen as the optimized formulation because it has satisfactory viscosity gelling capacity and ease of application into the olfactory route [40].

Table 9: Niosomal in situ nasal gel formulation of rivastigmine of various concentrations

\begin{tabular}{|c|c|c|c|c|c|c|c|}
\hline \multirow{2}{*}{$\begin{array}{l}\text { Batch } \\
\text { code }\end{array}$} & \multirow{2}{*}{$\begin{array}{l}\text { Carbopol 934P } \\
(\% \mathrm{w} / \mathrm{v})\end{array}$} & \multirow{2}{*}{$\begin{array}{l}\text { HPMC K4M } \\
(\% \mathrm{w} / \mathrm{v})\end{array}$} & \multirow{2}{*}{$\begin{array}{l}\text { Benzalkonium } \\
\text { Chloride }(\% \mathrm{w} / \mathrm{v})\end{array}$} & \multirow{2}{*}{$\begin{array}{l}\text { Gelling time } \\
\text { (min) }\end{array}$} & \multirow{2}{*}{$\begin{array}{l}\text { Gelling } \\
\text { capacity }\end{array}$} & \multicolumn{2}{|c|}{ Viscocity (mPa s at $15 \mathrm{rpm}$ ) } \\
\hline & & & & & & At pH 5 & At pH 6 \\
\hline ING1 & 0.1 & 0.2 & 1 & $12.2 \pm 0.23$ & - & $1345 \pm 0.13$ & $5760 \pm 0.11$ \\
\hline ING2 & 0.1 & 0.4 & 1 & $11.0 \pm 0.26$ & + & $1530 \pm 0.11$ & $6719 \pm 0.13$ \\
\hline ING3 & 0.1 & 0.6 & 1 & $10.4 \pm 0.32$ & +++ & $1650 \pm 0.16$ & $7832 \pm 0.32$ \\
\hline ING4 & 0.2 & 0.2 & 1 & $6.1 \pm 0.52$ & + & $1610 \pm 0.21$ & $6980 \pm 0.14$ \\
\hline ING5 & 0.2 & 0.4 & 1 & $6.3 \pm 0.34$ & + & $1830 \pm 0.22$ & $7340 \pm 0.23$ \\
\hline ING6 & 0.2 & 0.6 & 1 & $5.1 \pm 0.82$ & ++ & $2120 \pm 0.25$ & $7930 \pm 0.32$ \\
\hline ING7 & 0.3 & 0.2 & 1 & $5.3 \pm 0.44$ & +++ & $2430 \pm 0.42$ & $8120 \pm 0.42$ \\
\hline ING8 & 0.3 & 0.4 & 1 & $5.5 \pm 0.62$ & +++ & $2920 \pm 0.12$ & $8650 \pm 0.16$ \\
\hline ING9 & 0.3 & 0.6 & 1 & $5.7 \pm 0.24$ & +++ & $3430 \pm 0.43$ & $9135 \pm 0.28$ \\
\hline
\end{tabular}

mean \pm SD $(n=3)$, -No gelation, +gels slowly and dissolves, ++gelation immediate, remains for hours, +++-gelation immediate, remains for many hours.

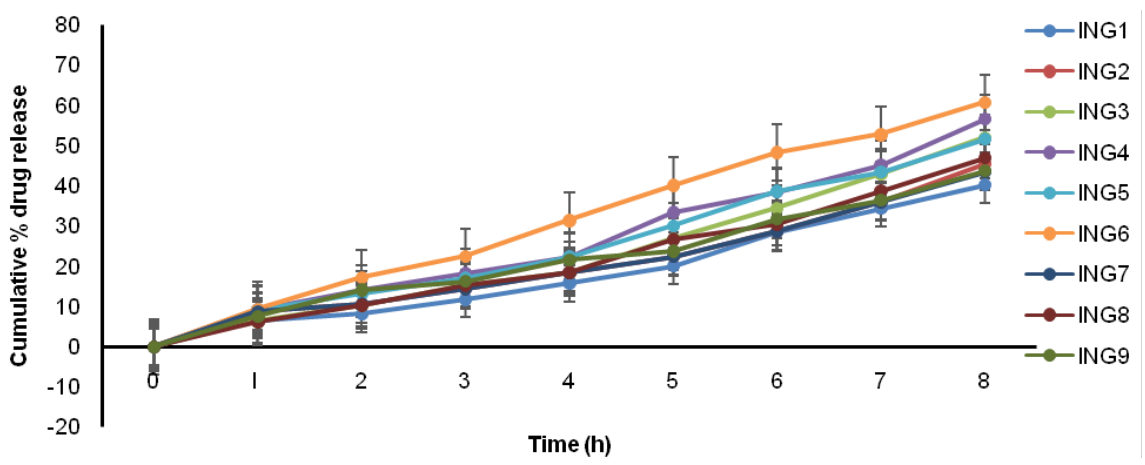

Fig. 16: Cumulative \% drug permeation of niosomal in situ nasal gel formulation

\section{In vitro permeation studies}

Fig. 16 shown the permeation-study results of nine different formulations. Of various batches ING6 showed maximum permeation of Rivastigmine (60.49\%) in 8 h. Plain Rivastigmine gel is a Carbopol 934P based gel signifying a simple, aqueous system with Rivastigmine dispersed in the matrix. Permeation studies of Rivastigmine from plain drug loaded gel determines the permeation properties of the drug. 
Fig. 17 shown the results of permeation study of ING6 showing the cumulative amount of drug released for the duration of 1-8 h. The flux (Jss) for ING6 was found to be $0.0831 \mathrm{mg} / \mathrm{cm}^{2} / \mathrm{h}$. The permeability coefficient (Kp) for ING6 was found to be $0.0145 \mathrm{~cm}^{2} / \mathrm{h}$ (table 10). The flux and permeability coefficient for formulation
ING6 were found to be higher, indicating that a niosomal $\mathrm{pH}$ induced in situ gel containing carbopol had a higher release of drug and permeability coefficient. As a result, nonionic surfactants found in niosomes work as a permeation enhancer, helping to improve penetration $[41,42]$

Table 10: Cumulative amount of drug released for the duration of 1-8 $\mathrm{h}$

\begin{tabular}{lll}
\hline Formulation & Flux (Jss) $\mathbf{~ m g} / \mathbf{c m}^{2} / \mathbf{h}$ & Permeability coefficient (Kp) $\mathbf{c m}^{2} / \mathbf{h}$ \\
\hline $\mathrm{NR} 1$ & $0.0831 \mathrm{mg} / \mathrm{cm}^{2} / \mathrm{h}$ & $0.0145 \mathrm{~cm}^{2} / \mathrm{h}$ \\
\hline
\end{tabular}

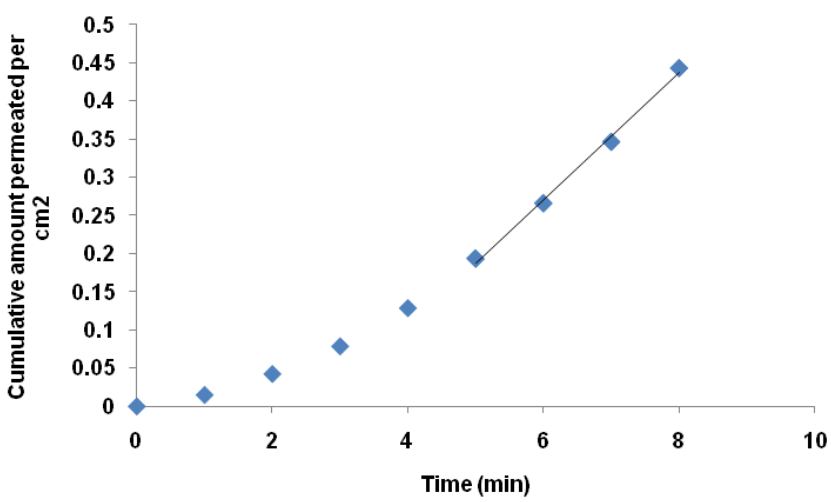

Fig. 17: Cumulative amount of drug permeation of optimized in situ gel formulation ING6

\section{CONCLUSION}

In the present study we have developed a mathematical model, optimized using $3^{2}$ factorial design using Design Expert software for better formulation of niosomes and also to understand the effect of various factors on particle size, entrapment efficiency and drug release at $8^{\text {th }}$ hour. Rivastigmine niosomes were prepared by thin film hydration technique and the best formulation NR1 is identified by the DOE approach and was evaluated for various parameters. Identifying the best optimized formulation, Rivastigmine loaded niosomal in situ nasal gel was prepared and proven to be a reliable method for obtaining a stable Rivastigmine niosomal gel. NR1 batch of Rivastigmine loaded niosome formulation has the best vesicle particle size, entrapment efficiency, and percentage drug release. SEM analysis demonstrates the production of spherical and smooth vesicles. The results of the zeta potential research show that the optimised niosomal formulation is resistant to aggregation.

Additionally the produced niosomes were effectively loaded in in situ gelling liquid compositions that had the requisite features in terms of clarity appearance homogeneity and consistency as well as gelling capacity at nasal $\mathrm{pH}$. In vitro drug permeation experiments show that using an appropriate niosomal gel formulation prolongs the efficasy of the drug. Thus niosomes outperformed standard gel formulations for intranasal medication delivery of Rivastigmine. Rivastigmine has been successfully developed as an intranasal in situ gel medication of administration for Alzheimer's disease.

\section{ACKNOWLEDGEMENT}

The authors are thankful to SRM College of Pharmacy, SRMIST, Kattankulathur, Tamilnadu, for providing their facilities and support for carrying out this research work and Dean, SRM College of Pharmacy, SRMIST for providing their services and support to complete the studies.

Institutional Animal Ethical Committee (IAEC Registration number 662/PO/RE/S/2002/CPCSEA Dated on 14/02/2020).

\section{FUNDING}

Nil

\section{ABBREVIATIONS}

RIV: Rivastigmine, AD: Alzhemier's Disease, BBB: Blood brain barrier, EE: Entrapment efficiency, SEM: Scanning Electron
Microscopy, TEM: Transmission electron microscopy, XRPD: X-Ray Powder Diffractometry, DSC: Differential scanning calorimetry, DOE: Design of Experiments, ANOVA: Analysis of Variance, Jss: Steady state flux, Kp: Permeation Coefficient, HPMC: Hydroxy propyl methyl cellulose, HLB: Hydrophilic-lipophilic balance; CPP: Critical packing parameter, rpm: revolution per minute.

\section{AUTHORS CONTRIBUTIONS}

All the author have contributed equally.

\section{CONFLICT OF INTERESTS}

Declared none

\section{REFERENCES}

1. Squire LR, Zola Morgan S. The medial temporal lobe memory system. Science 1991;253:1380-6.

2. Cummings JL, Cole G. Alzheimer disease. JAMA 2002;287:2335-8.

3. Hussain AA. Intranasal drug delivery. Adv Drug Delivery Rev 1998;29:39-4.

4. Barakat NS, Omar SA, Ahmed AAE. Carbamazepine uptake into rat brain following intra-olfactory transport. J Pharm Pharmacol 2005;58:63-72.

5. Dahlin M, Bergman U, Jansson B, Bjork E, Brittebo E. Transfer of dopamine in the olfactory pathway following nasal administration in mice. Pharm Res 2000;17:737-42.

6. Henriksson J, Tjalve H. Uptake of inorganic mercury in the olfactory bulbs via olfactory pathways in rats. Environ Res 1998;77:130-40.

7. Chou KJ, Donovan MD. Lidocaine distribution into the CNS following nasal and arterial delivery: a comparision of local sampling and microdialysis techniques. Int J Pharm 1998;171:53-61.

8. Eriksson C, Bergman U, Franzen A, Sjoblom M, Brittebo EB. Transfer of some carboxylic acids in the olfactory system following intranasal administration. J Drug Target 1997;7:131-42.

9. Prey WH, Liu J, Chen X, Thorne RG, Fawcett JR, Ala TA, et al. Delivery of $125 \mathrm{I}-\mathrm{NGF}$ to the brain via the olfactory route. Drug Delivery 1997;4:87-92.

10. Thorne RG, Emory CR, Ala TA, Frey WH. Quantitative analysis of the olfactory pathway for drug delivery to the brain. Brain Res 1995;692:278-82.

11. Illum L. Transport of drugs from the nasal cavity to the central nervous system. Eur J Pharm Sci 2000;11:1-18. 
12. Polinsky RJ. Clinical pharmacology of rivastigmine: a new generation acetyl choline esterase inhibitor for the treatment of Alzheimer's disease. Clin Ther 1998;20:634-7.

13. Subodh D, Amit J, Mehta SC, Pavan G, Sandeep J, Jagdish S. Niosomes: the ultimate drug carrier. Drug Inven Today 2010;2:72-7.

14. Uchegbu FI, Vyas PS. Non-ionic surfactant based vesicles (niosomes) in drug delivery. Int J Pharm 1998;33:172.

15. Rajalakshmi SV, Vinaya OG. Formulation development, evaluation and optimization of medicated lip rouge containing niosomal acyclovir for the management of recurrent herpes labialis. Int J Appl Pharm Sci 2017;9:21-7.

16. Lavanya B, Indira S, Srinivas P. Formulation and evaluation of ocular niosomal in situ gels of linezolid. Int J Pharm Sci Res 2014;5:1367-75.

17. Astrid Permatasari Isnan, Mahdi Jufri. Formulation of niosomal gel containing green tea extract (camellia sinensis l. kuntze) using thinlayer hydration. Int J Appl Pharm 2017;9 Suppl 1:38-43.

18. Sathali AAH, Rajalakshmi G. Evaluation of transdermal targeted niosomal drug delivery of terbinafine hydrochloride. Int J PharmTech Res 2010;2:2081-9.

19. Gayathri Devi S, Venkatesh, Udupa N. Niosomal sumatriptan succinate for nasal administration. Int J Pharm Sci 2010;62:480-1.

20. Hussain A, Dittert LW, Traboulsi A. Brain delivery of folic acid for the prevention of of Alzheimer's disease and stroke. US Patent 6369058B1; 2002.

21. Song J, Bi H, Xie X, Guo J, Wang X, Liu D. Preparation and evaluation of sinomenine hydrochloride in situ gel for uveitis treatment. Int Immunopharmacol 2013;17:99-107.

22. Cai Z, Song X, Sun F, Yang Z, Hou S, Liu Z. Formulation and evaluation of in situ gelling systems for intranasal administration of gastrodin. AAPS PharmSciTech 2011;12:1102-9.

23. Mahajan $\mathrm{H}$, Dinger S. Design and in vitro evaluation of nanoemulsion for nasal delivery of artemether. Ind J Nov Drug Delivery 2011;3:272-7.

24. Uchegbu IF, Double JA, Kelland LR, Turton JA, Florence AT. The activity of doxorubicin niosomes against an ovarian cancer cell line and three in vivo mouse tumour models. J Drug Target 1996;3:399-409.

25. Dahiya NK, Rao R, Nanda S. Preparation and characterization techniques in the niosomal vesicular systems-a review. J Pharm Biomed Sci 2011;5:1-8.

26. Tank Chintankumar J, Borkhataria Chetan H, Baria Ashok H, Patel Rakesh P, Tamizharasi S, Dipen K, et al. Formulation and evaluation of aceclofenac Loaded maltodextrin based proniosome. Int J Chem Tech Res 2009;1:567-73.

27. Girigoswami A, Das S, De S. Fluorescence and dynamic light scattering studies of niosomes-membrane mimetic systems. Spectrochim Acta A Mol Biomol Spectrosc 2006;64:859-66.
28. Kamel R, Basha M, Abdel Alim SH. Development of a novel vesicular system using a binary mixture of sorbitan monostearate and polyethylene glycol fatty acid esters for rectal delivery of rutin. J Lip Res 2013;23:28-36.

29. Guinedi AS, Mortada ND, Mansour S, Hathout RM. Preparation and evaluation of reverse-phase evaporation and multilamellar niosomes as ophthalmic carriers of acetazolamide. Int J Pharm 2005;306:71-82.

30. Rhodes D. Maltodextrin-based proniosomes. AAPS PharmSciTech 2001;3:1-8

31. Semple, Sean C Arcadio Chonn, Pieter R Cullis. Influence of cholesterol on the association of plasma proteins with liposomes. Biochem 1996;35:2521-5.

32. Kenneth J. Reversible gel forming composition for sustained delivery of bio-affecting substances, and methods of use. US Patent 5599534 A; 1997.

33. Dakhara Sanjay L, Anajwala Chetan C, Singhal Nirav A, Selote Vidula S. Phospholipids and non-ionic surfactants containing niosome is far better than liposome as ophthalmic dosage form. J Pharm Res 2010;3:2488-91.

34. Thomas L, Viswanad V. Formulation and optimization of clotrimazole-loaded proniosomal gel using 3 factorial design. Sci Pharm 2012;80:731-48.

35. Anjan D, Subrata C, Arup M, Jayanta C. Formulation and development of in situ gelling system for nasal administration for ondansetron hydrochloride by using pluronic F-127. J Pharm Pharm Sci 2013;2:52-61.

36. Srinivas S, Kumar YA, Hemanth A, Anitha M. Preparation and evaluation of niosomes containing aceclofenac. DJNB 2010;5:249-54.

37. Abdelbary GA, Aburahma MH. Oro-dental mucoadhesive proniosomal gel formulation loaded with lornoxicam for management of dental pain. J Liposome Res 2015;25:107-21.

38. Jain SP, Shah SP, Rajadhyaksha NS, Singh PS PP, Amin PD. In situ ophthalmic gel of ciprofloxacin hydrochloride for once a day sustained delivery. Drug Dev Ind Pharm 2008;34:445-52.

39. Ravouru N, Kondreddy P, Korakanchi D, Haritha M. Formulation and evaluation of niosomal nasal drug delivery system of folic acid for brain targeting. Curr Drug Discovery Technol 2013;10:270-82.

40. Shirsand SB, Para MS, Nagendrakumar D, Kanani KM, Keerthy D. Formulation and evaluation of Ketaconazole niosomal gel drug delivery system. Int J Pharm Invest 2012;2:201.

41. Akhtar N, Arkvanshi S, Bhattacharya SS, Verma A, Pathak K. Preparation and evaluation of a buflomedil hydrochloride niosomal patch for transdermal delivery. J Lip Res 2015;25:191-201.

42. Zhang Y, Zhang K, Wu Z, Guo T, Ye B, Lu M, et al. Evaluation of transdermal salidoside delivery using niosmes via in vitro cellular uptake. Int J Pharm 2015;478:138-46. 